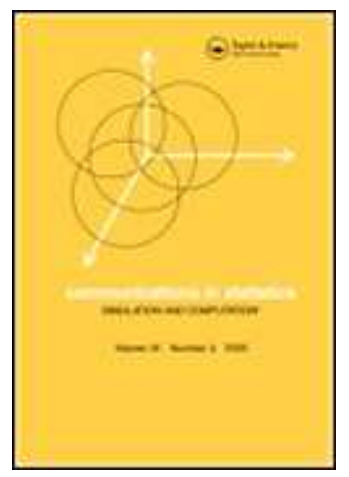

\title{
Confidence intervals for the weighted kappa coefficient of a binary diagnostic test
}

\begin{tabular}{|r|l|}
\hline Journal: & Communications in Statistics - Simulation and Computation \\
\hline Manuscript ID: & LSSP-2009-0045.R2 \\
\hline Manuscript Type: & Original Paper \\
\hline Author: & O5-May-2009 \\
\hline Complete List of Authors: & $\begin{array}{l}\text { Roldán Nofuentes, José Antonio; School of Medicine. University of } \\
\text { Granada, Biostatisitcs } \\
\text { Luna del Castillo, Juan de Dios; School of Medicine. University of } \\
\text { Granada, Biostatistics } \\
\text { Montero Alonso, Miguel Angel; School of Social Sciences. Campus } \\
\text { de Melilla. Univeristy of Granada, Statistics } \\
\text { Alvarado Castro, Victor Manuel; Autonomous University of } \\
\text { Guerrero, Mathematics }\end{array}$ \\
\hline Keywords: & \begin{tabular}{l} 
Binary test, Confidence interval, Weighted kappa coefficient \\
\hline Abstract:
\end{tabular} \\
$\begin{array}{l}\text { Sensitivity and specificity are classic parameters to assess the } \\
\text { performance of a binary diagnostic test. Another useful parameter } \\
\text { to measure the performance of a binary test is the weighted kappa } \\
\text { coefficient, which is a measure of the classificatory agreement } \\
\text { between the binary test and the gold standard. Various confidence } \\
\text { intervals are proposed for the weighted kappa coefficient when the } \\
\text { binary test and the gold standard are applied to all of the patients } \\
\text { in a random sample. The results have been applied to the diagnosis } \\
\text { of coronary artery disease. }\end{array}$ \\
\hline
\end{tabular}

\section{S ScholaroNE \\ Manuscript Central}




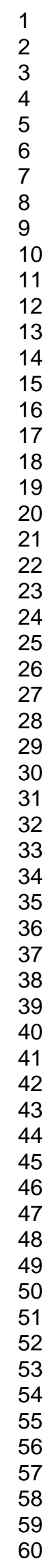




\title{
CONFIDENCE INTERVALS OF WEIGHTED KAPPA
}

\section{COEFFICIENT OF A BINARY DIAGNOSTIC TEST}

\author{
J.A. Roldán Nofuentes ${ }^{\mathrm{a}, 1}$, J.D. Luna del Castillo ${ }^{\mathrm{a}, 2}$, M.A. Montero Alonso ${ }^{\mathrm{b}, 3}$ and \\ V.M. Alvarado Castro ${ }^{\mathrm{c}, 4}$ \\ ${ }^{a}$ Biostatistics, School of Medicine, University of Granada, 18071, Spain \\ ${ }^{\mathrm{b}}$ Statistics, School of Social Sciences, Campus of Melilla, University of Granada, \\ 52005, Spain \\ ${ }^{c}$ Mathematics, School of Mathematics, Autonomous University of Guerrero, \\ 39070, Mexico \\ 1e-mail: jaroldan@ugr.es, ${ }^{2}$ e-mail: jdluna@ugr.es, ${ }^{3}$ e-mail: mmontero@ugr.es, \\ 4alvarado@cimat.mx
}

Summary. Sensitivity and specificity are classic parameters to assess the performance of a binary diagnostic test. Another useful parameter to measure the performance of a binary test is the weighted kappa coefficient, which is a measure of the classificatory agreement between the binary test and the gold standard. Various confidence intervals are proposed for the weighted kappa coefficient when the binary test and the gold standard are applied to all of the patients in a random sample. The results have been applied to the diagnosis of coronary artery disease.

Key words: Binary test; Confidence interval; Weighted kappa coefficient. 


\section{Introduction}

A diagnostic test is a test which is applied to a patient to determine the presence or absence of a determined disease. When the result of a diagnostic test is binary (positive or negative result), the accuracy of the diagnostic test is measured in terms of two parameters, sensitivity and specificity. Sensitivity is the probability of the result of the diagnostic test being positive given that the patient is diseased, and specificity is the probability of the result of the diagnostic test being negative given that the patient is not diseased. In order to obtain an unbiased estimator of sensitivity and specificity of the diagnostic test it is necessary to know the true disease status of each patient. The test through which the true disease status of each patient is known is called the gold Standard e.g a biopsy, a surgical operation etc. Other classic parameters to assess the performance of a binary diagnostic test are the positive and negative likelihood ratios and the positive and negative predictive values. The likelihood ratios quantify the increase in the knowledge of the disease after the application of the diagnostic test (Roldán Nofuentes and Luna del Castillo, 2007) and only depend on the sensitivity and specificity of the binary test. The predictive values represent the clinical accuracy of the diagnostic test and depend on the sensitivity and specificity of the diagnostic test and the disease prevalence. Therefore, there are various parameters which allow us to assess the performance of a binary diagnostic test. Bloch (1991) defined the weighted kappa coefficient of the risk of error of a binary test as a measure of the classificatory agreement between the diagnostic test and the gold standard, and deduced the asymptotic hypothesis tests to compare the weighted kappa coefficients of two binary tests subject to a paired design and case control design. In the study carried out by Kraemer et al (2002), one can observe a review of the use of the kappa coefficient in 
medical research. Donner et al (2003) developed a method to compare two dependent kappa coefficients. Roldán Nofuentes and Luna del Castillo $(2005,2007)$ have studied the estimation and comparison of the weighted kappa coefficients in the presence of partial disease verification. In this article, we study four confidence intervals for the weighted kappa coefficient when the diagnostic test and the gold standard are applied to all of the patients in a random sample. In Section 2, we study the weighted kappa coefficient of a binary test and its properties. In Section 3, four confidence intervals are proposed for the weighted kappa coefficient based on the asymptotic normality of the estimator of the weighted kappa coefficient and the asymptotic normality of the logit of this estimator and we also propose a bootstrap confidence interval. In Section 4, simulation experiments are carried out to study the performance of the confidence intervals proposed in Section 3. In Section 5, the results obtained have been applied to the study proposed by Weiner et al (1979) on the diagnosis of coronary disease, and in Section 6 the results obtained are discussed.

\section{Weighted kappa coefficient}

Let us consider a binary diagnostic test whose performance is assessed with in relation a gold standard. Let $T$ be the random variable which models the result of the diagnostic test, in such a way that $T=1$ when the result of the test is positive (indicating the provisional presence of the disease) and $T=0$ when the result of the test is negative (indicating the provisional absence of the disease); and let $D$ be the random variable which models the result of the gold standard in such a way that $D=1$ when the patient is diseased and $D=0$ when the patient does not have the disease. Let $S e=P(T=1 \mid D=1)$ and $S p=P(T=0 \mid D=0)$ be the sensitivity and the specificity of 
the diagnostic test respectively. Let $p=P(D=1)$ be the disease prevalence and $q=1-p$. Let us consider that $L$ is the loss or cost that occurs when erroneously classifying a diseased patient with the diagnostic test and that $L^{\prime}$ is the loss or cost that occurs when erroneously classifying a non-diseased patient. In Table 1 we show the frequencies obtained, along with the probabilities associated with each frequency and the losses, when the diagnostic test and the gold standard are applied to all of the patients in a random sample sized $n$.It is assumed that the losses $L$ and $L^{\prime}$ are 0 when a patient (diseased or non-diseased) is correctly classified with the diagnostic test. In terms of the previous probabilities and the losses, the expected loss observed when applying the diagnostic test (risk of error in the terminology of Bloch (1991)) is

$$
p(1-S e) L+q(1-S p) L^{\prime}
$$

and the expected random loss is

$$
p\{p(1-S e)+q S p\} L+q\{p S e+q(1-S p)\} L^{\prime} .
$$

The observed expected loss is the average loss which occurs when erroneously classifying a diseased or non-diseased patient with the diagnostic test, and its range of values varies from zero to infinite. The random expected loss is the expected loss which occurs when the diagnostic test and the gold standard are independent i.e. when $P(T=i \mid D=j)=P(T=i)$. In practice, both the observed expected loss and the random expected loss cannot be estimated since the losses $L$ and $L^{\prime}$ associated to an erroneous classification are unknown values. In terms of the observed expected loss and the random expected loss, the weighted kappa coefficient of a binary diagnostic test is defined as

$$
\kappa=\frac{\text { Random loss }- \text { Observed loss }}{\text { Random loss }}
$$


which is a measure of the relative discrepancy between the random expected loss and the observed expected loss. Substituting in equation (3) each loss with its expression it holds that the weighted kappa coefficient of the binary diagnostic test is

$$
\kappa_{c}=\frac{p q Y}{p\{p(1-S e)+q S p\} c+q\{p S e+q(1-S p)\}(1-c)}
$$

where $Y=S e+S p-1$ is the Youden index and $c=L /\left(L+L^{\prime}\right)$ is the weighting index.

When the loss $L$ is zero then $c=0$ and the weighted kappa coefficient is

$$
\kappa_{0}=\frac{S p-(1-Q)}{Q}=\frac{P P V-p}{1-p},
$$

when $P P V$ is the positive predictive value; and when the loss $L^{\prime}$ is zero then $c=1$ and the weighted kappa coefficient is

$$
\kappa_{1}=\frac{S e-Q}{1-Q}=\frac{N P V-(1-p)}{p}
$$

when $N P V$ is the negative predictive value. The weighted kappa coefficient $\kappa_{c}$ can be written in terms of $p, Q, \kappa_{0}$ and $\kappa_{1}$ as

$$
\kappa_{c}=\frac{p(1-Q) c \kappa_{1}+(1-p) Q(1-c) \kappa_{0}}{p(1-Q) c+(1-p) Q(1-c)}
$$

with $0<c<1$ and $Q=p S e+(1-p)(1-S p)$, and therefore the weighted kappa coefficient is a weighted average of $\kappa_{0}$ and $\kappa_{1}$. The weighting index varies between 0 and 1 and represents the relative loss between the false positives and the false negatives. In practice the $c$ index is unknown, but its values can be inferred depending on the objective for which the diagnostic test is going to be used. If the diagnostic test is going to be used as a screening test, there is a greater concern regarding the false negatives and the $c$ coefficient is greater than 0.5 ; if the diagnostic test is going to be used as a 
previous step before an intensive treatment, there is more concern about the false positives and the $c$ coefficient is lower than 0.5 ; and the $c$ index is 0.5 when the diagnostic test is used for a simple diagnosis. If in equation (4) $L=L^{\prime}$, then $c=0.5$ and $\kappa(0.5)$ is known as Cohen's kappa coefficient; if $L>L^{\prime}$ then $0.5<c<1$, and if $L^{\prime}>L$ then $0<c<0.5$. The weighted kappa coefficient of a binary test has the following properties: if the classificatory agreement between the binary test and the gold standard is perfect $(S e=S p=1)$ then the observed expected loss is 0 and $\kappa(c)=1$; if the sensitivity and the specificity are complementary $(S e=1-S p)$ then $\kappa_{c}=0$; if the random expected loss is greater than the observed expected loss then $\kappa_{c}>0$, and if the observed expected loss is greater than the random expected loss then $\kappa_{c}<0$ and the results of the diagnosis are interchanged, $T=1$ should be a negative result and $T=0$ should be a positive result; and the analysis should be limited only to the positive values of the weighted kappa coefficient. In Figures 1 and 2 we show how the weighted kappa coefficient varies according to the weighting index $c$ and the disease prevalence, for the same values of sensitivity and specificity. The weighted kappa coefficient is an increasing or decreasing function, depending on the disease prevalence, in the weighting index $c$.

Therefore, the weighted kappa coefficient of a binary diagnostic test is a valid measurement to assess the performance of a diagnostic test and measures the classificatory agreement or concordance between the diagnostic test and the gold standard when both are applied to the same group of patients. We then studied different confidence intervals for the weighted kappa coefficient. 


\section{Confidence intervals}

Let us consider the random variables $T$ and $D$ defined in the previous section. When the binary test and the gold standard are applied to all of the patients in a random sample sized $n$ we obtain Table 1 (observed frequencies). As the observed frequencies of Table 1 are the product of a multinomial distribution, substituting in equation (4) each parameter with its maximum likelihood estimator (MLE), through Zehna's Theorem, the MLE of the weighted kappa coefficient is

$$
\hat{\kappa}_{c}=\frac{s_{1} r+r_{0} s-s r}{n_{0} s c+n_{1} r(1-c)}
$$

with $0<c<1$, and applying the delta method (Serfling, 1980) the estimator of the variante of $\hat{\kappa}(c)$ is

$$
\begin{gathered}
\hat{\operatorname{Var}}\left(\hat{\kappa}_{c}\right)=\frac{n r}{s\left[n^{2}(1-c) r_{1}+n\left(c r_{0}-2(1-c) r_{1}\right) s_{0}+n\left\{r_{0}-(1-c) r_{1}\right\} s_{1}+s\left(s_{0} r_{1}-s_{1} r_{0}\right)\right]^{4}} \times \\
\left\{\left(s_{0} r_{1}-s_{1} r_{0}\right)^{2}\left[2(1-c) r_{1} n s-(1-c) r_{1} n^{2}+s\left(c\left(s_{0} r_{0}+2 s_{0} r_{1}+s_{1} r_{1}\right)-r_{1} s\right)\right]^{2}+\right. \\
\left.s_{1} s_{0} n r^{3}\left[(1-c) r_{1} n+s\left(c r-r_{1}\right)\right]^{2}+r_{1} r_{0} n s r^{2}\left[s_{1} r+c\left(s^{2}-s_{1} n\right)\right]^{2}\right\} .
\end{gathered}
$$

The demonstration can be seen in Appendix I. We now propose various confidence intervals for the weighted kappa coefficient of a binary diagnostic test.

\subsection{Wald type confidence interval}

Based on the asymptotic normality of $\left(\hat{\kappa}_{c}-\kappa_{c}\right) / \sqrt{\hat{\operatorname{Var}}\left(\hat{\kappa}_{c}\right)}$, the $100(1-\alpha) \%$ confidence interval for the weighted kappa coefficient is

$$
\hat{\kappa}_{c} \pm z_{1-\alpha / 2} \sqrt{\hat{\operatorname{Var}}\left(\hat{\kappa}_{c}\right)}
$$


where $z_{1-\alpha / 2}$ is the $100(1-\alpha / 2)$ th percentile of the standard normal distribution and $\hat{\operatorname{Var}}\left(\hat{\kappa}_{c}\right)$ is given by equation (9). This confidence interval can also be obtained with continuity correction, and the interval is

$$
\hat{\kappa}_{c} \pm\left(z_{1-\alpha / 2} \sqrt{\hat{\operatorname{Var}}\left(\hat{\kappa}_{c}\right)}+\frac{1}{2 n}\right) .
$$

\subsection{Logit confidence interval}

In the analysis of $2 \times 2$ tables, it frequently the case that the study of a parameter is not carried out directly on the parameter itself but on transformations of this parameter, such as the logit transformation. For example, for a binomial proportion it is possible to obtain a confidence interval based on the logit transformation of the proportion (Rubin and Schenker, 1987). For a weighted kappa coefficient, its analysis is limited only to the values between one and zero, and therefore the analysis is limited to the same values of a binomial proportion, so that the logit transformation is valid to study the weighted kappa coefficient. Based on the asymptotic normality of $\hat{\kappa}_{c}$, the logit transformation of $\hat{\kappa}_{c}, \log \left(\hat{\kappa}_{c} /\left(1-\hat{\kappa}_{c}\right)\right)$, shows a normal distribution with an average $\log \left(\kappa_{c} /\left(1-\kappa_{c}\right)\right)$. Thus, the $100(1-\alpha) \%$ confidence interval for $\log \left(\kappa_{c} /\left(1-\kappa_{c}\right)\right)$ is

$$
\operatorname{logit}\left(\hat{\kappa}_{c}\right) \pm z_{1-\alpha / 2} \sqrt{\hat{\operatorname{Var}}\left(\operatorname{logit}\left(\hat{\kappa}_{c}\right)\right)}
$$

Applying the delta method (Serfling, 1980), the estimator of the variance of the logit of $\hat{\kappa}_{c}$ is 


$$
\begin{gathered}
\hat{\operatorname{Var}}\left(\operatorname{logit}\left(\hat{\kappa}_{c}\right)\right)=\frac{1}{\left[r_{1} s-(1-c) r_{1} n-c\left(s_{0} r_{0}+2 s_{0} r_{1}+s_{1} r_{1}\right)\right]^{2}} \times \\
\left\{\frac{s_{1} s_{0} r^{2}\left[(1-c) r_{1} n+\left(c r-r_{1}\right) s\right]^{2}+r_{1} r_{0} s r\left[s_{1} n-s_{1} s+c\left(s^{2}-s_{1} n\right)\right]^{2}}{s\left(s_{0} r_{1}-s_{1} r_{0}\right)^{2}}+\right. \\
\frac{\left[2(1-c) r_{1} n s-(1-c) r_{1} n^{2}+s\left(c\left(s_{0} r_{0}+2 s_{0} r_{1}+s_{1} r_{1}\right)-r_{1} s\right)\right]}{n s r} .
\end{gathered}
$$

The demonstration can also be seen in Appendix I. Finally, the logit CI for the weighted kappa coefficient is

$$
\left(\frac{\exp \left\{\operatorname{logit}\left(\hat{\kappa}_{c}\right)-z_{1-\alpha / 2} \hat{\sigma}_{\text {logit }}\right\}}{1+\exp \left\{\operatorname{logit}\left(\hat{\kappa}_{c}\right)-z_{1-\alpha / 2} \hat{\sigma}_{\text {logit }}\right\}}, \frac{\exp \left\{\operatorname{logit}\left(\hat{\kappa}_{c}\right)+z_{1-\alpha / 2} \hat{\sigma}_{\text {logit }}\right\}}{1+\exp \left\{\operatorname{logit}\left(\hat{\kappa}_{c}\right)+z_{1-\alpha / 2} \hat{\sigma}_{\text {logit }}\right\}}\right)
$$

where $\hat{\sigma}_{\text {logit }}=\sqrt{\hat{\operatorname{Var}}\left(\operatorname{logit}\left(\hat{\kappa}_{c}\right)\right)}$.

\subsection{Bootstrap confidence interval}

The bootstrap confidence interval is calculated using 1000 replicas, each made by sampling $n$ subjects with replacement from the original $n$ subjects, and calculating for each one of them an estimator of the weighted kappa coefficient given by equation (8). As the estimator of the weighted kappa coefficient the average is proponed, in the thousand replicas of these estimators, and the overall confidence interval is calculated using the bias-corrected CI (Efron and Tibshirani, 1993).

\section{Simulation study}

In order to study the performance of the confidence intervals proposed in the previous section, Monte Carlo simulation experiments were carried out which consisted of the generation of 10000 random samples of multinomial distributions sized 100, 200, 300, 
400500 and 1000, and whose probabilities were calculated from the probabilities that appear in Table 1 (probabilities). These experiments were designed from equation (7) in the following way. As prevalence values we took the values $0.10,0.50$ and 0.90 , as values of $\kappa_{0}$ and $\kappa_{1}$ we took the values $0.1,0.2, \ldots, 0.9$, and as values of the weighting index $c$ we took $0.1,0.5$ and 0.9 . Once the values of $p, \kappa_{0}$ and $\kappa_{1}$ are set, the values of sensitivity and specificity were calculated from equations (5) and (6), and the value of $\kappa_{c}$ was calculated from equation (7). Therefore, the probabilities of the multinomial distributions from the values of the weighted kappa coefficients and without setting the values of sensitivity and specificity. For each one of the 10000 random samples of a multinomial distribution the Wald interval (with and without continuity correction) and the logit interval were calculated, and for each of 10000 random samples we calculated the coverage and the average length of each one of these three intervals. Regarding the Bootstrap interval, for each one of the 10000 samples we have generated 1000 random bootstrap samples and for each one these 1000 samples we have calculated the biascorrected interval and later we have calculated the coverage and the average length. The simulation experiments were designed in such a way that in all of the samples the marginal totals are all greater than zero (since if this were not the case, it would not be possible to estimate the weighted kappa coefficient). For all of the simulation study the level of confidence has been set at $95 \%$. In Tables 2, 3, 4 and 5 we show some of the results obtained for $\kappa_{c}$ equal to $0.2,0.4,0.6$ and 0.8 respectively. From the results of the simulation experiments the following conclusions are obtained in general terms. For samples of at least 200 individuals the coverage of the logit interval has a better performance than the rest of the intervals: once the coverage has reached $95 \%$, the coverage of the interval has fewer fluctuations around this value than the rest of the 
intervals. Regarding the performance of the intervals with small samples (sized 100), in general the performance of the intervals depends on the value of the weighted kappa coefficient: for very low values $\left(\kappa_{c}=0.2\right)$ the logit interval has a good coverage although in some cases the average length is a little larger than that of the rest of the intervals; for values of $\kappa_{c}$ equal to 0.4 and 0.6 , the Wald interval without continuity correction and the bootstrap interval have a good performance; and for very high values of the weighted kappa coefficient $\left(\kappa_{c}=0.8\right)$, the bootstrap interval has a better coverage than the rest of the intervals, although its average length is a little greater than that of the rest of the intervals. Regarding the Wald confidence interval with continuity correction, the continuity correction does not have an important effect on the performance of the interval and the performance of this interval is very similar to the Wald interval without correction.

\section{Application}

The results obtained were applied to the study of Weiner et al (1979) on the diagnosis of coronary artery disease, using as a diagnostic test an exercise test and as a gold standard a coronary arteriography. In Table 6 we show the results obtained by Weiner et al for males according to three different symptoms (definitive angina, probable angina and nonischemic pain), where the variable $T$ models the result of the exercise test and the variable $D$ models the result of the coronary arteriography. In Table 7 we show the estimated values and the confidence intervals for the weighted kappa coefficient for different values of the weighted index. In the case of males with definite angina or probable angina it holds that if the exercise test is used before an intensive treatment 
$(0<c<0.5)$ then the weighted kappa coefficient takes an intermediate value so that the classificatory agreement between the exercise test and the arteriography is moderate (Landis and Koch, 1977); nevertheless, if the exercise test is used as a screening test $(0.5<c<1)$, the weighted kappa coefficient takes low-intermediate values depending on the value of the weighted index, so that the classificatory agreement between the exercise test and the arteriography varies between regular and moderate. In the case of males with nonischemic pain, if the diagnostic test is used before an intensive treatment $(0<c<0.5)$, then the classificatory agreement between the exercise test and the arteriography varies between slight and regular depending on the value of the weighted index; if the test is used as a screening test $(0.5<c<1)$, the classificatory agreement between the exercise test and the arteriography varies between slight, regular and moderate, depending on the weighted index.

\section{Discussion}

The weighted kappa coefficient of a diagnostic test is a measure of the classificatory agreement between the binary test and the gold standard, and is a parameter which depends on the sensitivity and the specificity of the binary test, on the disease prevalence and on the medical costs of an erroneous classification of a diseased or nondiseased patient with the binary test. Depending on the disease prevalence, this parameter cannot be estimated if the sample is a case-control one (unless the value for the disease prevalence is known from other studies). Regarding the medical costs, these cannot be estimated, and therefore the choice of the value of the weighting index will depend on the clinical use of the binary test: if the binary test is used as a previous step 
to an intensive treatment then $0<c<0.5$, if the binary test is used as a screening test then $0.5<c<1$ and if the binary test is used for the simple diagnosis of a disease then $c=0.5$.

In this four confidence intervals are studied for the weighted kappa coefficient of a binary diagnostic test when the diagnostic test and the gold standard are applied to all of the patients in a random sample, of which three intervals are based on the asymptotic normality of the estimator of the weighted kappa coefficient (Wald interval with and without continuity correction and logit interval) and a fourth interval based on the bootstrap method. Monte Carlo simulation experiments have been carried out to study the performance, measured in terms of coverage and average length, of these confidence intervals to $95 \%$. From the results of the simulation experiments it holds that, in general terms, and for values of weighted kappa coefficients which are common in clinical practice, when the samples are small $(n=100)$ the Wald type interval without continuity correction and the Bootstrap interval have a good performance; whilst for large samples $(n \geq 200)$ the logit interval shows a better performance than the rest of the intervals. This better performance of the logit interval in large samples $(n \geq 200)$ must be due to the fact that the distribution of the logit of $\hat{\kappa}_{c}$ is less asymmetrical than the distribution of $\hat{\kappa}_{c}$ and of $\hat{\kappa}_{c B}$.

Furthermore, in order to estimate weighted kappa coefficient it is necessary for all of the marginal totals $\left(s, r, n_{1}\right.$ and $n_{0}$ ) of the table of observed frequencies to be greater than zero, since if one of these frequencies is zero it is not possible to estimate the weighted kappa coefficient nor to calculate any of the confidence intervals proposed in 
Section 3. When one of the marginal totals is zero, the problem can be solved in different ways:

1). Defining the estimated weighted kappa coefficient as zero, and taking a sufficiently large sample so that there are no zeros in the marginal totals.

2). Performing a case-control design in two stages. In the first stage, two samples are selected, one of $s$ diseased patients and another one of $r$ non-diseased patients, where $s$ and $r$ are set $a$ priori; and estimating the prevalence $p$. In the second stage, estimating the sensitivity from the sample of diseased patients and estimating the specificity from the sample of non-diseased patients. Finally, estimating the weighted kappa coefficient from the estimated values of prevalence, sensitivity and specificity.

3). Not setting any sample size a priori and taking patients until the sample size is greater than a pre-set value and all of the marginal totals are greater than zero. Although the method that is proposed in this study to solve the problem of estimating the weighted kappa coefficient is based on the conditioning in the totals of the rows in a sample sized $n$ (set a priori), and not in any of the alternatives previously mentioned, the problem of the samples with a marginal total equal to zero is not a major problem, since these samples are infrequent. In the simulation experiments carried out in Section 4 , we have counted the number of samples in which any marginal total was zero, finding that for samples of 100 the percentage of "defective" samples (with at least one marginal total equal to zero) was on average around 5\%, for samples of 200 the average percentage was less than $1 \%$ and for samples of 300 it was lower than $0.5 \%$, and was almost zero for larger samples. 
The solution of the problem of estimation posed in this study is based on the conditioning in the totals of the rows. Another way of solving this problem is from the unconditional point of view. In this situation, the estimators of sensitivity and specificity and, consequently the weighted kappa coefficient, are biased (Kraemer, 1992, pp 34-40), and the bias of each one of them depends on the sample size. When the sample size is large enough, the estimators are approximately unbiased and their estimated variances are approximately equal to those given in appendix I, and it is also verified that $\operatorname{Cov}(\hat{S} e, \hat{S} p)=0$ (Bennett, 1983). Therefore, from the unconditional point of view, when the sample size is large, the problem of estimation of the weighted kappa coefficient is solved in a similar way to the solution conditioning in the totals of rows.

\section{Acknowledgements}

This research was supported by the Department for Innovation, Science and Business of the Autonomous Government of Andalusia, Spain, grant number FQM-01459. We thank the editor and the referee for their helpful comments that improved the quality of the paper.

\section{Appendix I}

The data $\left(s_{1}, s_{0}, r_{1}, r_{0}\right)$ from Table 1 are the product of a multinomial distribution with probabilities $(p S e, p(1-S e),(1-p)(1-S p),(1-p) S p)$. In this table of frequencies the only fixed value is the value is the value of sample size $n$, so that conditioning in the totals of the rows $s_{1}$ has a binomial distribution with parameters $s$ and $S e$ and $r_{0}$ has a binomial distribution with parameters $r$ and Sp. Applying the properties of the binomial 
distribution, the data $\left(s_{1}, s_{0}\right)$ and $\left(r_{1}, r_{0}\right)$ can be treated as independent samples and consequently $\operatorname{Cov}(S e, S p)=0$. Moreover, the sensitivity and the specificity do not depend on the disease prevalence, but on the intrinsic properties of the diagnostic test to distinguish between diseased patients and non-diseased patients and, therefore, $\operatorname{Cov}(\operatorname{Se}, p)=\operatorname{Cov}(S p, p)=0$.

Therefore, conditioning in the totals of the rows it holds that the sensitivity, the specificity and the prevalence are binomial proportions, and their estimators are $\hat{S} e=\frac{s_{1}}{s}, \hat{S} p=\frac{r_{0}}{r}$ and $\hat{p}=\frac{s}{n}$ and their estimated variances are $\hat{\operatorname{Var}}(\hat{S} e)=\frac{\hat{S} e(1-\hat{S} e)}{s}$, $\hat{\operatorname{Var}}(\hat{S} p)=\frac{\hat{S} p(1-\hat{S} p)}{r}$ and $\hat{\operatorname{Var}}(\hat{p})=\frac{\hat{p} \hat{q}}{n}$ respectively, with $s_{1}$ and $r_{0}$ being over zero. Therefore, for the estimation of the weighted kappa coefficient it is necessary to impose that all the marginal frequencies $\left(s, r, n_{1}\right.$ and $\left.n_{0}\right)$ are over zero. Applying the delta method (Serfling, 1980) the variance of weighted kappa coefficient is

$$
\operatorname{Var}\left(\hat{\kappa}_{c}\right)=\left(\frac{\partial \kappa_{c}}{\partial S e}\right)^{2} \operatorname{Var}(S e)+\left(\frac{\partial \kappa_{c}}{\partial S p}\right)^{2} \operatorname{Var}(S p)+\left(\frac{\partial \kappa_{c}}{\partial p}\right)^{2} \operatorname{Var}(p)
$$

Carrying out the operations and substituting each parameter with its MLE we obtain equation (9). Regarding the variance of the logit of the weighted kappa coefficient, this is calculated in a similar way to the previous one:

$$
\begin{gathered}
\operatorname{Var}\left(\operatorname{logit}\left(\hat{\kappa}_{c}\right)\right)= \\
\left(\frac{\partial \operatorname{logit}\left(\kappa_{c}\right)}{\partial S e}\right)^{2} \operatorname{Var}(S e)+\left(\frac{\partial \operatorname{logit}\left(\kappa_{c}\right)}{\partial S p}\right)^{2} \operatorname{Var}(S p)+\left(\frac{\partial \operatorname{logit}\left(\kappa_{c}\right)}{\partial p}\right)^{2} \operatorname{Var}(p) .
\end{gathered}
$$




\section{References}

Bennett, B.M., 1983. Further results on indices of diagnostic screening. Biometrical Journal 24, 59-62.

Bloch, D.A., 1997. Comparing two diagnostic tests against the same "gold standard" in the same sample. Biometrics 53, 73-85.

Donner, A., Shoukri, M.M., Klar, N., Bartfay, E., 2000. Testing the equality of two dependent kappa statistics. Statistics in Medicine 19, 373-387.

Efron, B., Tibshirani, R.J., 1993. An Introduction to the Bootstrap. Chapman and Hall, New York.

Kraemer, H.C., 1992. Evaluating medical tests. Objective and quantitative guidelines. Sage Publications, Newbury Park.

Kraemer, H.C., Periyakoil, V.S., Noda, A., 2002. Kappa coefficients in medical research. Statistics in Medicine 2, 2109-2129.

Landis, J.R., Koch., G.G., 1977. The measurement of observer agreement for categorical data. Biometrics 33, 159-174.

Roldán Nofuentes, J.A., Luna del Castillo, J.D., 2006. Comparing two binary diagnostic tests in the presence of verification bias. Computational Statistics and Data Analysis $50,1551-1564$.

Roldán Nofuentes, J.A., Luna del Castillo, J.D., 2007. Risk of error and kappa coefficient of a binary diagnostic test in presence of partial verification. Journal of Applied Statistics 34, 887-898.

Rubin D.B., Schenker, N., 1987. Logit-based interval estimation for binomial data using the Jefreys prior. Sociological Methodology 17, 131-144. 
Serfling, R.J., 1980. Approximation theorems of mathematical statistics. John Wiley and Sons, New York.

Weiner, D.A., Ryan, T.J., McCabe, C.H., Kennedy, J.W., Schloss, M., Tristani, F., Chaitman, B.R., Fisher, L.D., 1979. Exercise stress testing. Correlations among history of angina, ST-segmant response and prevalence of coronary-artery disease in the coronary artery surgery study (CASS). The New England Journal of Medicine $301,230-235$. 
Table 1. Observed frequencies, probabilities and losses.

\begin{tabular}{|c|c|c|c|}
\hline \multicolumn{4}{|c|}{ Observed frequencies } \\
\hline & $T=1$ & $T=0$ & Total \\
\hline$D=1$ & $s_{1}$ & $s_{0}$ & $s$ \\
\hline$D=0$ & $r_{1}$ & $r_{0}$ & $r$ \\
\hline Total & $n_{1}$ & $n_{0}$ & $n$ \\
\hline \multicolumn{4}{|c|}{ Probabilities } \\
\hline & $T=1$ & $T=0$ & Total \\
\hline$D=1$ & $p S e$ & $p(1-S e)$ & $p$ \\
\hline$D=0$ & $q(1-S p)$ & $q S p$ & $q=1-p$ \\
\hline Total & $Q=p S e+q(1-S p)$ & $1-Q=p(1-S e)+q S p$ & 1 \\
\hline \multicolumn{4}{|c|}{ Losses } \\
\hline & $T=1$ & $T=0$ & Total \\
\hline$D=1$ & 0 & $L$ & $L$ \\
\hline$D=0$ & $L^{\prime}$ & 0 & $L^{\prime}$ \\
\hline Total & $L^{\prime}$ & $L$ & $L+L^{\prime}$ \\
\hline
\end{tabular}


Table 2. Coverages and lengths of weight kappa coefficient confidence intervals $\left(\kappa_{c}=0.2\right)$.

\begin{tabular}{|c|c|c|c|c|c|c|c|c|}
\hline \multicolumn{9}{|c|}{$p=0.10 \quad \kappa_{0}=0.2 \quad \kappa_{1}=0.2 \quad c=0.1 \quad S e=0.28 \quad S p=0.92$} \\
\hline & \multicolumn{2}{|c|}{ Wald CI } & \multicolumn{2}{|c|}{ Waldcc CI } & \multicolumn{2}{|c|}{ Logit CI } & \multicolumn{2}{|c|}{ Bootstrap CI } \\
\hline$n$ & Cov. & Long. & Cov. & Long. & Cov. & Long. & Cov. & Long. \\
\hline 100 & 0.950 & 0.559 & 0.954 & 0.569 & 0.959 & 0.615 & 0.941 & 0.532 \\
\hline 200 & 0.938 & 0.396 & 0.942 & 0.401 & 0.955 & 0.415 & 0.924 & 0.386 \\
\hline 300 & 0.937 & 0.324 & 0.939 & 0.327 & 0.958 & 0.345 & 0.926 & 0.320 \\
\hline 400 & 0.936 & 0.282 & 0.940 & 0.284 & 0.967 & 0.292 & 0.932 & 0.280 \\
\hline 500 & 0.935 & 0.251 & 0.935 & 0.253 & 0.969 & 0.257 & 0.930 & 0.251 \\
\hline 1000 & 0.946 & 0.179 & 0.947 & 0.180 & 0.958 & 0.180 & 0.946 & 0.179 \\
\hline \multicolumn{9}{|c|}{$p=0.10 \quad \kappa_{0}=0.2 \quad \kappa_{1}=0.2 \quad c=0.5 \quad S e=0.28 \quad S p=0.92$} \\
\hline & \multicolumn{2}{|c|}{ Wald CI } & \multicolumn{2}{|c|}{ Waldcc CI } & \multicolumn{2}{|c|}{ Logit CI } & \multicolumn{2}{|c|}{ Bootstrap CI } \\
\hline$n$ & Cov. & Long. & Cov. & Long. & Cov. & Long. & Cov. & Long. \\
\hline 100 & 0.960 & 0.533 & 0.971 & 0.543 & 0.954 & 0.600 & 0.943 & 0.487 \\
\hline 200 & 0.940 & 0.381 & 0.944 & 0.386 & 0.960 & 0.410 & 0.928 & 0.364 \\
\hline 300 & 0.932 & 0.312 & 0.934 & 0.316 & 0.963 & 0.336 & 0.922 & 0.305 \\
\hline 400 & 0.940 & 0.272 & 0.943 & 0.274 & 0.963 & 0.284 & 0.935 & 0.268 \\
\hline 500 & 0.936 & 0.244 & 0.937 & 0.246 & 0.961 & 0.251 & 0.927 & 0.242 \\
\hline 1000 & 0.944 & 0.174 & 0.945 & 0.175 & 0.956 & 0.175 & 0.941 & 0.174 \\
\hline \multicolumn{9}{|c|}{$0.9 S e=0.28 S p$} \\
\hline & \multicolumn{2}{|c|}{ Wald CI } & \multicolumn{2}{|c|}{ Waldcc CI } & \multicolumn{2}{|c|}{ Logit CI } & \multicolumn{2}{|c|}{ Bootstrap CI } \\
\hline$n$ & Cov. & Long. & Cov. & Long. & Cov. & Long. & Cov. & Long. \\
\hline 100 & 0.960 & 0.560 & 0.964 & 0.570 & 0.960 & 0.615 & 0.961 & 0.517 \\
\hline 200 & 0.947 & 0.395 & 0.953 & 0.400 & 0.967 & 0.416 & 0.931 & 0.378 \\
\hline 300 & 0.943 & 0.325 & 0.945 & 0.328 & 0.965 & 0.343 & 0.932 & 0.319 \\
\hline 400 & 0.930 & 0.282 & 0.934 & 0.284 & 0.962 & 0.294 & 0.923 & 0.279 \\
\hline 500 & 0.945 & 0.251 & 0.946 & 0.253 & 0.963 & 0.257 & 0.938 & 0.250 \\
\hline 1000 & 0.948 & 0.179 & 0.950 & 0.180 & 0.957 & 0.179 & 0.947 & 0.179 \\
\hline \multicolumn{9}{|c|}{$p=0.50 \quad \kappa_{0}=0.2 \quad \kappa_{1}=0.2 \quad c=0.1 S e=0.60 \quad S p=0.60$} \\
\hline & \multicolumn{2}{|c|}{ Wald CI } & \multicolumn{2}{|c|}{ Waldec CI } & & $\mathrm{CI}$ & Boo & $\mathrm{p} \mathrm{CI}$ \\
\hline$n$ & Cov. & Long. & Cov. & Long. & Cov. & Long. & Cov. & Long. \\
\hline 100 & 0.970 & 0.392 & 0.974 & 0.402 & 0.953 & 0.435 & 0.963 & 0.364 \\
\hline 200 & 0.951 & 0.277 & 0.954 & 0.282 & 0.963 & 0.281 & 0.938 & 0.270 \\
\hline 300 & 0.943 & 0.226 & 0.945 & 0.230 & 0.956 & 0.232 & 0.936 & 0.224 \\
\hline 400 & 0.944 & 0.196 & 0.947 & 0.199 & 0.961 & 0.198 & 0.943 & 0.196 \\
\hline 500 & 0.946 & 0.176 & 0.948 & 0.178 & 0.965 & 0.177 & 0.945 & 0.175 \\
\hline 1000 & 0.947 & 0.124 & 0.950 & 0.125 & 0.954 & 0.124 & 0.945 & 0.124 \\
\hline & & $p=0.10$ & $0=0.2$ & $0.2 c=0.5$ & $S e=0$. & $S p=0.60$ & & \\
\hline & & CI & & & & $\mathrm{CI}$ & Boo & $\mathrm{p} \mathrm{CI}$ \\
\hline$n$ & Cov. & Long. & Cov. & Long. & Cov. & Long. & Cov. & Long. \\
\hline 100 & 0.960 & 0.379 & 0.968 & 0.389 & 0.940 & 0.430 & 0.947 & 0.345 \\
\hline 200 & 0.944 & 0.270 & 0.955 & 0.275 & 0.952 & 0.275 & 0.935 & 0.262 \\
\hline 300 & 0.947 & 0.221 & 0.951 & 0.224 & 0.962 & 0.226 & 0.940 & 0.218 \\
\hline 400 & 0.942 & 0.192 & 0.944 & 0.194 & 0.964 & 0.194 & 0.941 & 0.190 \\
\hline 500 & 0.937 & 0.171 & 0.942 & 0.173 & 0.951 & 0.172 & 0.937 & 0.171 \\
\hline 1000 & 0.948 & 0.121 & 0.950 & 0.122 & 0.959 & 0.122 & 0.945 & 0.121 \\
\hline & & $p=0.10$ & $0=0.2$ & $0.2 c=0.1$ & $S e=0.6$ & $S p=0.60$ & & \\
\hline & & CI & & & & $\mathrm{CI}$ & Boo & $\mathrm{p} \mathrm{CI}$ \\
\hline$n$ & Cov. & Long. & Cov. & Long. & Cov. & Long. & Cov. & Long. \\
\hline 100 & 0.970 & 0.392 & 0.974 & 0.402 & 0.955 & 0.436 & 0.964 & 0.363 \\
\hline 200 & 0.950 & 0.278 & 0.956 & 0.283 & 0.959 & 0.282 & 0.939 & 0.271 \\
\hline 300 & 0.950 & 0.226 & 0.955 & 0.230 & 0.954 & 0.231 & 0.945 & 0.225 \\
\hline 400 & 0.951 & 0.196 & 0.953 & 0.199 & 0.961 & 0.198 & 0.947 & 0.196 \\
\hline 500 & 0.947 & 0.175 & 0.950 & 0.177 & 0.957 & 0.177 & 0.944 & 0.175 \\
\hline 1000 & 0.958 & 0.124 & 0.959 & 0.125 & 0.959 & 0.124 & 0.958 & 0.124 \\
\hline
\end{tabular}


Table 3. Coverages and lengths of weight kappa coefficient confidence intervals $\left(\kappa_{c}=0.4\right)$.

\begin{tabular}{|c|c|c|c|c|c|c|c|c|}
\hline \multicolumn{9}{|c|}{$p=0.10 \quad \kappa_{0}=0.4 \quad \kappa_{1}=0.4 \quad c=0.1 S e=0.46 S p=0.94$} \\
\hline & \multicolumn{2}{|c|}{ Wald CI } & \multicolumn{2}{|c|}{ Waldcc CI } & \multicolumn{2}{|c|}{ Logit CI } & \multicolumn{2}{|c|}{ Bootstrap CI } \\
\hline$n$ & Cov. & Long. & Cov. & Long. & Cov. & Long. & Cov. & Long. \\
\hline 100 & 0.914 & 0.609 & 0.920 & 0.619 & 0.977 & 0.561 & 0.915 & 0.617 \\
\hline 200 & 0.933 & 0.437 & 0.935 & 0.442 & 0.967 & 0.415 & 0.934 & 0.443 \\
\hline 300 & 0.923 & 0.358 & 0.928 & 0.362 & 0.953 & 0.345 & 0.927 & 0.361 \\
\hline 400 & 0.936 & 0.311 & 0.939 & 0.313 & 0.950 & 0.302 & 0.936 & 0.313 \\
\hline 500 & 0.940 & 0.278 & 0.941 & 0.280 & 0.954 & 0.271 & 0.942 & 0.279 \\
\hline 1000 & 0.944 & 0.197 & 0.946 & 0.198 & 0.951 & 0.195 & 0.944 & 0.197 \\
\hline \multicolumn{9}{|c|}{$p=0.10 \quad \kappa_{0}=0.6 \quad \kappa_{1}=0.3 \quad c=0.5 \quad S e=0.34 S p=0.98$} \\
\hline & \multicolumn{2}{|c|}{ Wald CI } & \multicolumn{2}{|c|}{ Waldcc CI } & \multicolumn{2}{|c|}{ Logit CI } & \multicolumn{2}{|c|}{ Bootstrap CI } \\
\hline$n$ & Cov. & Long. & Cov. & Long. & Cov. & Long. & Cov. & Long. \\
\hline 100 & 0.926 & 0.618 & 0.927 & 0.628 & 0.980 & 0.567 & 0.926 & 0.625 \\
\hline 200 & 0.935 & 0.444 & 0.938 & 0.449 & 0.968 & 0.423 & 0.939 & 0.448 \\
\hline 300 & 0.944 & 0.367 & 0.946 & 0.371 & 0.969 & 0.354 & 0.945 & 0.371 \\
\hline 400 & 0.941 & 0.318 & 0.943 & 0.321 & 0.962 & 0.309 & 0.943 & 0.320 \\
\hline 500 & 0.940 & 0.285 & 0.940 & 0.287 & 0.955 & 0.278 & 0.940 & 0.287 \\
\hline 1000 & 0.950 & 0.202 & 0.952 & 0.203 & 0.957 & 0.200 & 0.947 & 0.203 \\
\hline \multicolumn{9}{|c|}{$p=0.10 k$} \\
\hline & \multicolumn{2}{|c|}{ Wald CI } & \multicolumn{2}{|c|}{ Waldcc CI } & \multicolumn{2}{|c|}{ Logit CI } & \multicolumn{2}{|c|}{ Bootstrap CI } \\
\hline$n$ & Cov. & Long. & Cov. & Long. & Cov. & Long. & Cov. & Long. \\
\hline 100 & 0.937 & 0.479 & 0.942 & 0.489 & 0.977 & 0.455 & 0.933 & 0.479 \\
\hline 200 & 0.946 & 0.341 & 0.947 & 0.346 & 0.967 & 0.330 & 0.945 & 0.343 \\
\hline 300 & 0.946 & 0.279 & 0.948 & 0.283 & 0.960 & 0.273 & 0.946 & 0.280 \\
\hline 400 & 0.941 & 0.243 & 0.944 & 0.245 & 0.951 & 0.238 & 0.939 & 0.243 \\
\hline 500 & 0.946 & 0.217 & 0.948 & 0.219 & 0.951 & 0.214 & 0.948 & 0.217 \\
\hline 1000 & 0.944 & 0.154 & 0.945 & 0.155 & 0.949 & 0.152 & 0.943 & 0.154 \\
\hline \multicolumn{9}{|c|}{$p=0.50 \quad \kappa_{0}=0.4 \quad \kappa_{1}=0.4 \quad c=0.1 S e=0.70 \quad S p=0.70$} \\
\hline & \multicolumn{2}{|c|}{ Wald CI } & \multicolumn{2}{|c|}{ Waldcc CI } & & $\mathrm{CI}$ & Boo & p CI \\
\hline$n$ & Cov. & Long. & Cov. & Long. & Cov. & Long. & Cov. & Long. \\
\hline 100 & 0.947 & 0.382 & 0.951 & 0.392 & 0.975 & 0.367 & 0.945 & 0.382 \\
\hline 200 & 0.944 & 0.271 & 0.947 & 0.276 & 0.952 & 0.265 & 0.943 & 0.271 \\
\hline 300 & 0.945 & 0.221 & 0.948 & 0.225 & 0.956 & 0.218 & 0.945 & 0.221 \\
\hline 400 & 0.945 & 0.192 & 0.947 & 0.194 & 0.951 & 0.190 & 0.944 & 0.192 \\
\hline 500 & 0.957 & 0.172 & 0.960 & 0.174 & 0.958 & 0.170 & 0.955 & 0.172 \\
\hline 1000 & 0.955 & 0.122 & 0.959 & 0.123 & 0.957 & 0.121 & 0.956 & 0.122 \\
\hline & & $p=0.50$ & $=0.3$ & $0.6 c=$ & $S e=0$. & $S p=0.53$ & & \\
\hline & & $\mathrm{CI}$ & & $\mathrm{CI}$ & & $\mathrm{CI}$ & Boo & p CI \\
\hline$n$ & Cov. & Long. & Cov. & Long. & Cov. & Long. & Cov. & Long. \\
\hline 100 & 0.951 & 0.335 & 0.955 & 0.345 & 0.975 & 0.325 & 0.950 & 0.336 \\
\hline 200 & 0.942 & 0.239 & 0.947 & 0.244 & 0.956 & 0.234 & 0.943 & 0.239 \\
\hline 300 & 0.950 & 0.195 & 0.954 & 0.198 & 0.956 & 0.193 & 0.950 & 0.195 \\
\hline 400 & 0.944 & 0.169 & 0.948 & 0.171 & 0.949 & 0.167 & 0.942 & 0.169 \\
\hline 500 & 0.943 & 0.151 & 0.946 & 0.153 & 0.948 & 0.150 & 0.944 & 0.151 \\
\hline 1000 & 0.947 & 0.107 & 0.950 & 0.108 & 0.948 & 0.107 & 0.949 & 0.107 \\
\hline & & $p=0.50$ & $=0.1$ & $0.6 c=$ & $S e=0$ & $S p=0.23$ & & \\
\hline & & CI & $\mathrm{Wc}$ & CI & & $\mathrm{CI}$ & Boo & p CI \\
\hline$n$ & Cov. & Long. & Cov. & Long. & Cov. & Long. & Cov. & Long. \\
\hline 100 & 0.959 & 0.530 & 0.961 & 0.540 & 0.976 & 0.501 & 0.945 & 0.508 \\
\hline 200 & 0.937 & 0.377 & 0.938 & 0.382 & 0.956 & 0.364 & 0.931 & 0.373 \\
\hline 300 & 0.938 & 0.310 & 0.941 & 0.313 & 0.957 & 0.301 & 0.937 & 0.308 \\
\hline 400 & 0.931 & 0.268 & 0.932 & 0.271 & 0.949 & 0.263 & 0.931 & 0.268 \\
\hline 500 & 0.945 & 0.241 & 0.947 & 0.243 & 0.953 & 0.237 & 0.946 & 0.240 \\
\hline 1000 & 0.956 & 0.171 & 0.957 & 0.172 & 0.957 & 0.169 & 0.954 & 0.171 \\
\hline
\end{tabular}


Table 4. Coverages and lengths of weight kappa coefficient confidence intervals $\left(\kappa_{c}=0.6\right)$.

\begin{tabular}{|c|c|c|c|c|c|c|c|c|}
\hline \multicolumn{9}{|c|}{$p=0.10 \quad \kappa_{0}=0.6 \quad \kappa_{1}=0.6 \quad c=0.1 S e=0.64 S p=0.96$} \\
\hline & \multicolumn{2}{|c|}{ Wald CI } & \multicolumn{2}{|c|}{ Waldcc CI } & \multicolumn{2}{|c|}{ Logit $\mathrm{CI}$} & \multicolumn{2}{|c|}{ Bootstrap CI } \\
\hline$n$ & Cov. & Long. & Cov. & Long. & Cov. & Long. & Cov. & Long. \\
\hline 100 & 0.925 & 0.590 & 0.927 & 0.600 & 0.986 & 0.535 & 0.937 & 0.612 \\
\hline 200 & 0.926 & 0.417 & 0.930 & 0.422 & 0.959 & 0.396 & 0.931 & 0.424 \\
\hline 300 & 0.930 & 0.342 & 0.932 & 0.346 & 0.954 & 0.330 & 0.933 & 0.345 \\
\hline 400 & 0.944 & 0.298 & 0.945 & 0.301 & 0.961 & 0.290 & 0.945 & 0.301 \\
\hline 500 & 0.940 & 0.266 & 0.941 & 0.268 & 0.948 & 0.261 & 0.940 & 0.268 \\
\hline 1000 & 0.941 & 0.189 & 0.943 & 0.190 & 0.949 & 0.187 & 0.943 & 0.190 \\
\hline \multicolumn{9}{|c|}{$p=0.10 \quad \kappa_{0}=0.6 \quad \kappa_{1}=0.6 \quad c=0.5 \quad S e=0.64 \quad S p=0.96$} \\
\hline & \multicolumn{2}{|c|}{ Wald CI } & \multicolumn{2}{|c|}{ Waldcc CI } & \multicolumn{2}{|c|}{ Logit CI } & \multicolumn{2}{|c|}{ Bootstrap CI } \\
\hline$n$ & Cov. & Long. & Cov. & Long. & Cov. & Long. & Cov. & Long. \\
\hline 100 & 0.932 & 0.530 & 0.941 & 0.540 & 0.984 & 0.488 & 0.939 & 0.553 \\
\hline 200 & 0.942 & 0.376 & 0.945 & 0.381 & 0.963 & 0.360 & 0.947 & 0.384 \\
\hline 300 & 0.933 & 0.306 & 0.937 & 0.309 & 0.949 & 0.297 & 0.938 & 0.310 \\
\hline 400 & 0.952 & 0.265 & 0.955 & 0.268 & 0.964 & 0.259 & 0.955 & 0.268 \\
\hline 500 & 0.956 & 0.238 & 0.958 & 0.240 & 0.965 & 0.234 & 0.958 & 0.240 \\
\hline 1000 & 0.954 & 0.168 & 0.957 & 0.169 & 0.956 & 0.167 & 0.954 & 0.169 \\
\hline \multicolumn{9}{|c|}{$9 S e=0.64 S p=1$} \\
\hline & \multicolumn{2}{|c|}{ Wald CI } & \multicolumn{2}{|c|}{ Waldcc CI } & \multicolumn{2}{|c|}{ Logit CI } & \multicolumn{2}{|c|}{ Bootstrap CI } \\
\hline$n$ & Cov. & Long. & Cov. & Long. & Cov. & Long. & Cov. & Long. \\
\hline 100 & 0.926 & 0.594 & 0.927 & 0.604 & 0.985 & 0.538 & 0.931 & 0.615 \\
\hline 200 & 0.923 & 0.419 & 0.931 & 0.424 & 0.959 & 0.398 & 0.929 & 0.426 \\
\hline 300 & 0.936 & 0.344 & 0.937 & 0.347 & 0.959 & 0.331 & 0.937 & 0.347 \\
\hline 400 & 0.947 & 0.298 & 0.948 & 0.300 & 0.963 & 0.290 & 0.948 & 0.300 \\
\hline 500 & 0.945 & 0.268 & 0.947 & 0.270 & 0.954 & 0.262 & 0.950 & 0.269 \\
\hline 1000 & 0.945 & 0.189 & 0.946 & 0.190 & 0.952 & 0.187 & 0.944 & 0.190 \\
\hline \multicolumn{9}{|c|}{$p=0.50 \quad \kappa_{0}=0.6 \quad \kappa_{1}=0.6 \quad c=0.1 \quad S e=0.80 \quad S p=0.80$} \\
\hline & \multicolumn{2}{|c|}{ Wald CI } & \multicolumn{2}{|c|}{ Waldec CI } & & CI & Boo & $\mathrm{p} \mathrm{CI}$ \\
\hline$n$ & Cov. & Long. & Cov. & Long. & Cov. & Long. & Cov. & Long. \\
\hline 100 & 0.930 & 0.350 & 0.934 & 0.360 & 0.953 & 0.338 & 0.929 & 0.350 \\
\hline 200 & 0.936 & 0.250 & 0.941 & 0.255 & 0.952 & 0.245 & 0.936 & 0.249 \\
\hline 300 & 0.941 & 0.205 & 0.945 & 0.208 & 0.945 & 0.202 & 0.941 & 0.204 \\
\hline 400 & 0.955 & 0.178 & 0.958 & 0.180 & 0.957 & 0.176 & 0.953 & 0.177 \\
\hline 500 & 0.941 & 0.159 & 0.946 & 0.161 & 0.948 & 0.157 & 0.945 & 0.158 \\
\hline 1000 & 0.950 & 0.112 & 0.953 & 0.113 & 0.953 & 0.112 & 0.954 & 0.112 \\
\hline & & $p=0.50$ & $=0.6 \quad$ & $0.6 c=0.5$ & $S e=0.8$ & $S p=0.80$ & & \\
\hline & & $\mathrm{CI}$ & & $\mathrm{CI}$ & & $\mathrm{CI}$ & Boo & p CI \\
\hline$n$ & Cov. & Long. & Cov. & Long. & Cov. & Long. & Cov. & Long. \\
\hline 100 & 0.926 & 0.311 & 0.938 & 0.321 & 0.955 & 0.302 & 0.932 & 0.312 \\
\hline 200 & 0.945 & 0.221 & 0.955 & 0.226 & 0.952 & 0.217 & 0.948 & 0.221 \\
\hline 300 & 0.953 & 0.181 & 0.954 & 0.184 & 0.961 & 0.179 & 0.955 & 0.181 \\
\hline 400 & 0.955 & 0.157 & 0.957 & 0.159 & 0.960 & 0.155 & 0.956 & 0.157 \\
\hline 500 & 0.953 & 0.140 & 0.954 & 0.142 & 0.957 & 0.139 & 0.953 & 0.140 \\
\hline 1000 & 0.947 & 0.099 & 0.950 & 0.100 & 0.948 & 0.099 & 0.948 & 0.099 \\
\hline & & $p=0.50$ & $=0.6 \mathrm{r}$ & $0.6 c=0.9$ & $S e=0$. & $S p=0.80$ & & \\
\hline & & $\mathrm{CI}$ & & & & CI & Boo & $\mathrm{p} \mathrm{CI}$ \\
\hline$n$ & Cov. & Long. & Cov. & Long. & Cov. & Long. & Cov. & Long. \\
\hline 100 & 0.941 & 0.351 & 0.946 & 0.361 & 0.968 & 0.339 & 0.940 & 0.351 \\
\hline 200 & 0.943 & 0.250 & 0.948 & 0.255 & 0.951 & 0.245 & 0.943 & 0.250 \\
\hline 300 & 0.948 & 0.204 & 0.952 & 0.208 & 0.955 & 0.202 & 0.948 & 0.204 \\
\hline 400 & 0.947 & 0.177 & 0.951 & 0.180 & 0.953 & 0.176 & 0.946 & 0.177 \\
\hline 500 & 0.949 & 0.159 & 0.953 & 0.161 & 0.953 & 0.157 & 0.949 & 0.158 \\
\hline 1000 & 0.950 & 0.112 & 0.954 & 0.113 & 0.952 & 0.112 & 0.950 & 0.112 \\
\hline
\end{tabular}


Table 5. Coverages and lengths of weight kappa coefficient confidence intervals $\left(\kappa_{c}=0.8\right)$.

\begin{tabular}{|c|c|c|c|c|c|c|c|c|}
\hline \multicolumn{9}{|c|}{$p=0.10 \kappa_{0}=0.9 \quad \kappa_{1}=0.4 \quad c=0.1 S e=0.43 S p=0.995$} \\
\hline & \multicolumn{2}{|c|}{ Wald CI } & \multicolumn{2}{|c|}{ Waldcc CI } & \multicolumn{2}{|c|}{ Logit CI } & \multicolumn{2}{|c|}{ Bootstrap CI } \\
\hline$n$ & Cov. & Long. & Cov. & Long. & Cov. & Long. & Cov. & Long. \\
\hline 100 & 0.977 & 0.699 & 0.983 & 0.709 & 0.986 & 0.607 & 0.976 & 0.754 \\
\hline 200 & 0.986 & 0.441 & 0.987 & 0.446 & 0.970 & 0.422 & 0.985 & 0.466 \\
\hline 300 & 0.986 & 0.334 & 0.987 & 0.338 & 0.972 & 0.329 & 0.989 & 0.348 \\
\hline 400 & 0.971 & 0.275 & 0.973 & 0.278 & 0.971 & 0.273 & 0.975 & 0.284 \\
\hline 500 & 0.964 & 0.241 & 0.966 & 0.243 & 0.966 & 0.240 & 0.971 & 0.246 \\
\hline 1000 & 0.935 & 0.164 & 0.936 & 0.165 & 0.946 & 0.164 & 0.940 & 0.166 \\
\hline \multicolumn{9}{|c|}{$p=0.10 \quad \kappa_{0}=0.8 \quad \kappa_{1}=0.8 \quad c=0.5 \quad S e=0.82 S p=0.98$} \\
\hline & \multicolumn{2}{|c|}{ Wald CI } & \multicolumn{2}{|c|}{ Waldcc CI } & \multicolumn{2}{|c|}{ Logit CI } & \multicolumn{2}{|c|}{ Bootstrap CI } \\
\hline$n$ & Cov. & Long. & Cov. & Long. & Cov. & Long. & Cov. & Long. \\
\hline 100 & 0.976 & 0.434 & 0.978 & 0.444 & 0.972 & 0.422 & 0.977 & 0.468 \\
\hline 200 & 0.932 & 0.286 & 0.938 & 0.291 & 0.960 & 0.286 & 0.937 & 0.296 \\
\hline 300 & 0.940 & 0.232 & 0.947 & 0.236 & 0.961 & 0.233 & 0.944 & 0.237 \\
\hline 400 & 0.932 & 0.200 & 0.934 & 0.203 & 0.954 & 0.201 & 0.933 & 0.204 \\
\hline 500 & 0.938 & 0.179 & 0.939 & 0.181 & 0.952 & 0.179 & 0.938 & 0.181 \\
\hline 1000 & 0.935 & 0.126 & 0.937 & 0.127 & 0.948 & 0.126 & 0.935 & 0.127 \\
\hline \multicolumn{9}{|c|}{$p=0.10 k$} \\
\hline & \multicolumn{2}{|c|}{ Wald CI } & \multicolumn{2}{|c|}{ Waldcc CI } & \multicolumn{2}{|c|}{ Logit CI } & \multicolumn{2}{|c|}{ Bootstrap CI } \\
\hline$n$ & Cov. & Long. & Cov. & Long. & Cov. & Long. & Cov. & Long. \\
\hline 100 & 0.979 & 0.454 & 0.990 & 0.464 & 0.971 & 0.434 & 0.978 & 0.459 \\
\hline 200 & 0.977 & 0.284 & 0.979 & 0.289 & 0.968 & 0.282 & 0.976 & 0.290 \\
\hline 300 & 0.963 & 0.221 & 0.965 & 0.224 & 0.966 & 0.220 & 0.968 & 0.224 \\
\hline 400 & 0.947 & 0.188 & 0.951 & 0.191 & 0.962 & 0.188 & 0.949 & 0.190 \\
\hline 500 & 0.947 & 0.168 & 0.949 & 0.170 & 0.955 & 0.168 & 0.951 & 0.169 \\
\hline 1000 & 0.941 & 0.119 & 0.944 & 0.120 & 0.945 & 0.119 & 0.943 & 0.119 \\
\hline \multicolumn{9}{|c|}{$p=0.50 \quad \kappa_{0}=0.9 \quad \kappa_{1}=0.4 \quad c=0.1 S e=0.58 \quad S p=0.97$} \\
\hline & \multicolumn{2}{|c|}{ Wald CI } & \multicolumn{2}{|c|}{ Waldcc CI } & & $\mathrm{CI}$ & Boc & p CI \\
\hline$n$ & Cov. & Long. & Cov. & Long. & Cov. & Long. & Cov. & Long. \\
\hline 100 & 0.976 & 0.286 & 0.981 & 0.296 & 0.977 & 0.283 & 0.975 & 0.286 \\
\hline 200 & 0.951 & 0.187 & 0.959 & 0.192 & 0.960 & 0.187 & 0.952 & 0.188 \\
\hline 300 & 0.929 & 0.152 & 0.936 & 0.156 & 0.949 & 0.153 & 0.933 & 0.152 \\
\hline 400 & 0.930 & 0.132 & 0.936 & 0.135 & 0.938 & 0.132 & 0.933 & 0.132 \\
\hline 500 & 0.943 & 0.119 & 0.947 & 0.121 & 0.954 & 0.119 & 0.944 & 0.119 \\
\hline 1000 & 0.943 & 0.084 & 0.944 & 0.085 & 0.945 & 0.084 & 0.942 & 0.084 \\
\hline & & $p=0.50$ & $=0.8$ & $0.8 c=$ & $S e=0$ & $S p=0.90$ & & \\
\hline & & $\mathrm{CI}$ & & $\mathrm{CI}$ & & $\mathrm{CI}$ & Boc & $\mathrm{pCI}$ \\
\hline$n$ & Cov. & Long. & Cov. & Long. & Cov. & Long. & Cov. & Long. \\
\hline 100 & 0.933 & 0.275 & 0.926 & 0.285 & 0.958 & 0.278 & 0.935 & 0.275 \\
\hline 200 & 0.923 & 0.197 & 0.928 & 0.202 & 0.952 & 0.198 & 0.923 & 0.197 \\
\hline 300 & 0.933 & 0.162 & 0.940 & 0.165 & 0.957 & 0.162 & 0.932 & 0.161 \\
\hline 400 & 0.940 & 0.141 & 0.944 & 0.143 & 0.949 & 0.141 & 0.939 & 0.140 \\
\hline 500 & 0.940 & 0.126 & 0.943 & 0.128 & 0.951 & 0.126 & 0.940 & 0.126 \\
\hline 1000 & 0.943 & 0.090 & 0.946 & 0.091 & 0.948 & 0.090 & 0.941 & 0.089 \\
\hline & & $p=0.50$ & $=0.4$ & $0.9 c=$ & $S e=0$ & $S p=0.58$ & & \\
\hline & & CI & & $\mathrm{CI}$ & & $\mathrm{CI}$ & Boc & $\mathrm{p} \mathrm{CI}$ \\
\hline$n$ & Cov. & Long. & Cov. & Long. & Cov. & Long. & Cov. & Long. \\
\hline 100 & 0.978 & 0.288 & 0.982 & 0.298 & 0.976 & 0.288 & 0.977 & 0.290 \\
\hline 200 & 0.951 & 0.188 & 0.954 & 0.193 & 0.959 & 0.188 & 0.953 & 0.189 \\
\hline 300 & 0.942 & 0.153 & 0.948 & 0.156 & 0.952 & 0.153 & 0.944 & 0.153 \\
\hline 400 & 0.937 & 0.133 & 0.941 & 0.135 & 0.942 & 0.133 & 0.941 & 0.133 \\
\hline 500 & 0.939 & 0.119 & 0.942 & 0.121 & 0.941 & 0.119 & 0.938 & 0.119 \\
\hline 1000 & 0.949 & 0.084 & 0.950 & 0.085 & 0.952 & 0.084 & 0.949 & 0.084 \\
\hline
\end{tabular}


Table 6. Data from the study of Weiner et al.

\begin{tabular}{cccc}
\hline \multicolumn{4}{c}{ Definitive angina } \\
\hline$D=1$ & 473 & $T=0$ & Total \\
$D=0$ & 22 & 44 & 554 \\
\hline Total & 495 & 125 & 66 \\
\hline \multicolumn{4}{c}{ Probable angina } \\
\hline \multicolumn{5}{c}{$T=1$} & $T=0$ & Total \\
\hline$D=1$ & 313 & 102 & 415 \\
$D=0$ & 47 & 132 & 179 \\
\hline Total & 360 & 234 & 594 \\
\hline & Nonischemic pain & \\
\hline & $T=1$ & $T=0$ & Total \\
\hline$D=1$ & 29 & 25 & 54 \\
$D=0$ & 46 & 151 & 197 \\
\hline Total & 75 & 176 & 251 \\
\hline \hline
\end{tabular}


Table 7. Results of the study of Weiner et al.

\begin{tabular}{|c|c|c|c|c|}
\hline \multicolumn{5}{|c|}{ Definitive angina } \\
\hline$c$ & $\hat{\kappa}_{c}$ & Wald CI & Logit CI & Bootstrap CI \\
\hline 0.1 & 0.527 & $(0.405,0.643)$ & $(0.408,0.639)$ & $(0.404,0.640)$ \\
\hline 0.2 & 0.462 & $(0.367,0.584)$ & $(0.370,0.584)$ & $(0.366,0.581)$ \\
\hline 0.3 & 0.412 & $(0.334,0.538)$ & $(0.338,0.539)$ & $(0.333,0.535)$ \\
\hline 0.4 & 0.371 & $(0.305,0.500)$ & $(0.310,0.502)$ & $(0.304,0.497)$ \\
\hline 0.5 & 0.338 & $(0.280,0.467)$ & $(0.285,0.471)$ & $(0.279,0.465)$ \\
\hline 0.6 & 0.310 & $(0.257,0.439)$ & $(0.264,0.444)$ & $(0.257,0.438)$ \\
\hline 0.7 & 0.287 & $(0.238,0.415)$ & $(0.245,0.420)$ & $(0.238,0.414)$ \\
\hline 0.8 & 0.267 & $(0.221,0.393)$ & $(0.229,0.399)$ & $(0.221,0.393)$ \\
\hline 0.9 & 0.249 & $(0.207,0.374)$ & $(0.214,0.380)$ & $(0.206,0.374)$ \\
\hline \multicolumn{5}{|c|}{ Probable angina } \\
\hline$c$ & $\hat{\boldsymbol{\kappa}}_{c}$ & Wald CI & Logit CI & Bootstrap CI \\
\hline 0.1 & 0.543 & $(0.453,0.626)$ & $(0.452,0.624)$ & $(0.452,0.624)$ \\
\hline 0.2 & 0.523 & $(0.434,0.596)$ & $(0.434,0.594)$ & $(0.433,0.593)$ \\
\hline 0.3 & 0.504 & $(0.415,0.569)$ & $(0.416,0.569)$ & $(0.414,0.566)$ \\
\hline 0.4 & 0.486 & $(0.396,0.546)$ & $(0.398,0.546)$ & $(0.395,0.543)$ \\
\hline 0.5 & 0.470 & $(0.379,0.526)$ & $(0.380,0.526)$ & $(0.377,0.523)$ \\
\hline 0.6 & 0.454 & $(0.362,0.507)$ & $(0.364,0.508)$ & $(0.360,0.505)$ \\
\hline 0.7 & 0.440 & $(0.346,0.491)$ & $(0.348,0.492)$ & $(0.344,0.489)$ \\
\hline 0.8 & 0.426 & $(0.331,0.476)$ & $(0.333,0.477)$ & $(0.329,0.474)$ \\
\hline 0.9 & 0.413 & $(0.317,0.462)$ & $(0.319,0.464)$ & $(0.315,0.460)$ \\
\hline \multicolumn{5}{|c|}{ Nonischemic pain } \\
\hline$c$ & $\hat{\kappa}_{c}$ & Wald CI & Logit CI & Bootstrap CI \\
\hline 0.1 & 0.340 & $(0.110,0.343)$ & $(0.131,0.363)$ & $(0.106,0.346)$ \\
\hline 0.2 & 0.351 & $(0.116,0.354)$ & $(0.137,0.374)$ & $(0.112,0.356)$ \\
\hline 0.3 & 0.354 & $(0.123,0.367)$ & $(0.144,0.385)$ & $(0.119,0.368)$ \\
\hline 0.4 & 0.377 & $(0.130,0.380)$ & $(0.150,0.398)$ & $(0.126,0.380)$ \\
\hline 0.5 & 0.391 & $(0.137,0.395)$ & $(0.158,0.412)$ & $(0.133,0.394)$ \\
\hline 0.6 & 0.406 & $(0.144,0.412)$ & $(0.165,0.428)$ & $(0.140,0.410)$ \\
\hline 0.7 & 0.423 & $(0.152,0.431)$ & $(0.173,0.447)$ & $(0.147,0.429)$ \\
\hline 0.8 & 0.441 & $(0.159,0.453)$ & $(0.181,0.468)$ & $(0.155,0.451)$ \\
\hline 0.9 & 0.460 & $(0.165,0.478)$ & $(0.188,0.493)$ & $(0.162,0.477)$ \\
\hline
\end{tabular}


Figure 1. Weighted kappa coefficients for $S e=0.80, S p=0.75$ and different values of prevalence.

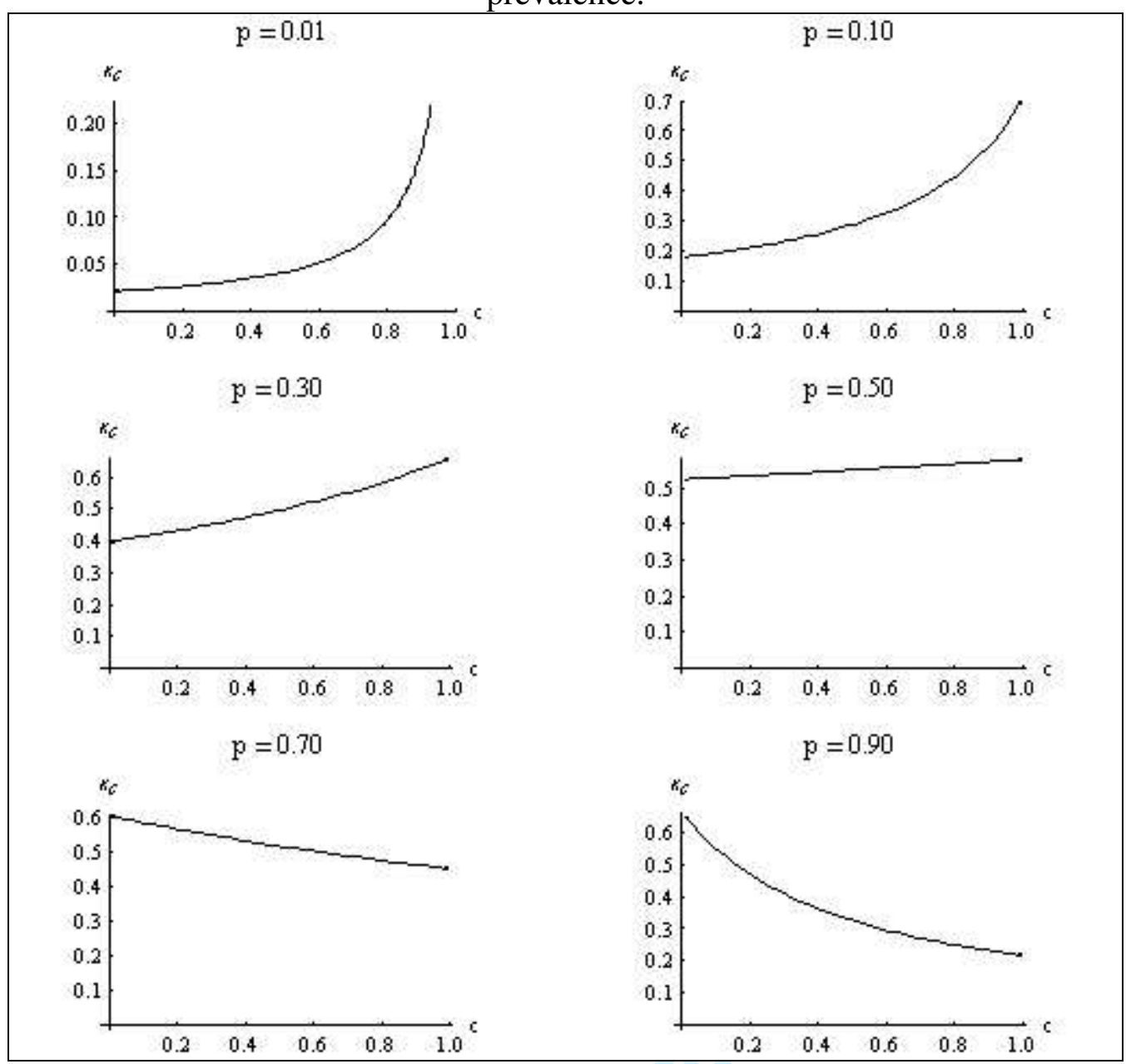


Figure 2. Weighted kappa coefficients for $S e=0.75, S p=0.95$ and different values of prevalence.

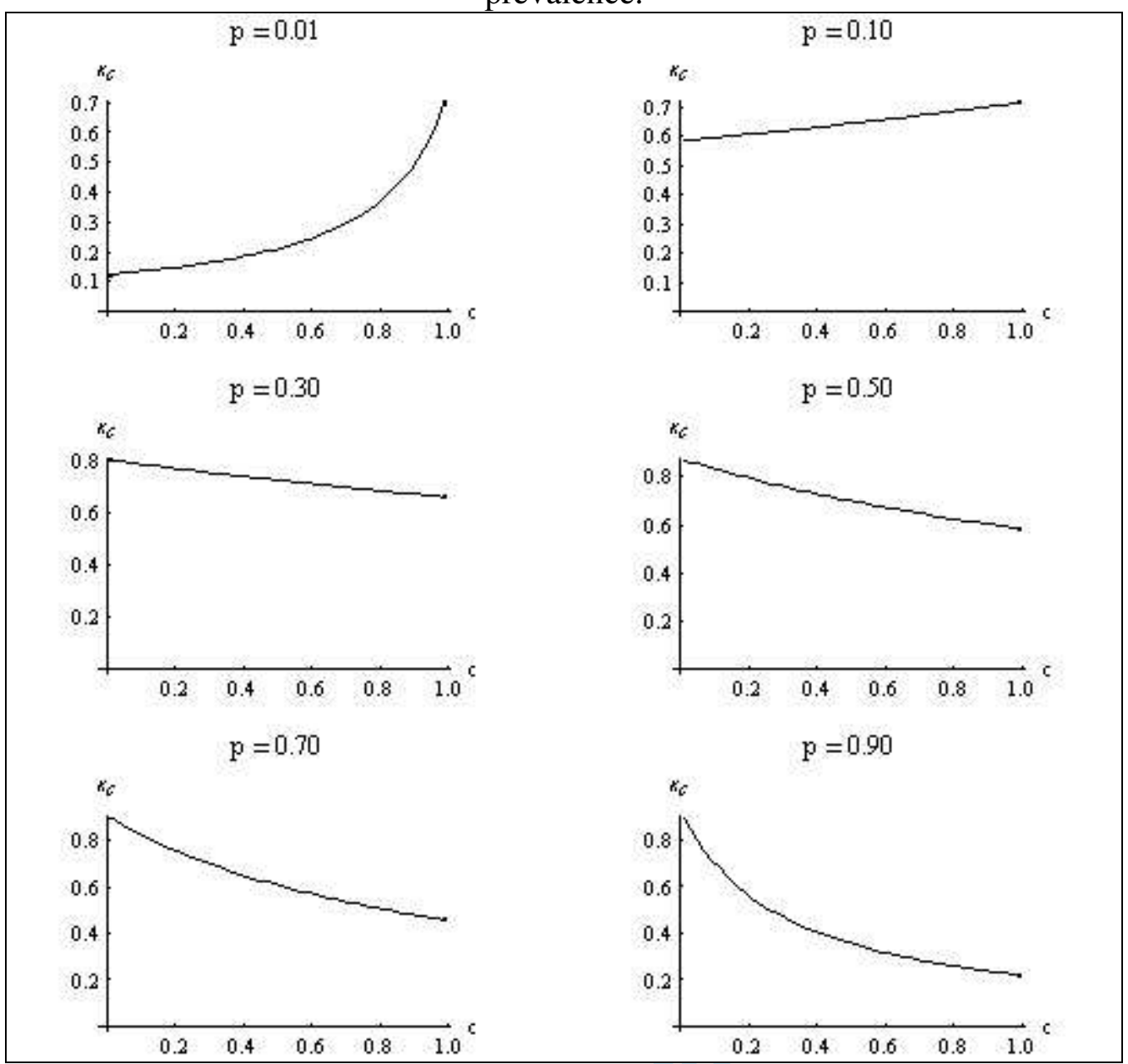

